\title{
SUMOylation of HSP27 regulates PKM2 to promote esophageal squamous cell carcinoma progression
}

\author{
XIAO ZHANG ${ }^{1,2^{*}}$, TAO LIU ${ }^{3 *}$, SHUTAO ZHENG $^{1,2}$, QING LIU $^{1,2}$, TONGXUE SHEN $^{1,2}$, \\ XIUJUAN HAN ${ }^{1,2}$, QIQI ZHANG ${ }^{1,2}$, LIFEI YANG ${ }^{4}$ and XIAOMEI LU ${ }^{1,2,5^{*}}$ \\ ${ }^{1}$ Clinical Medical Research Institute, First Affiliated Hospital of Xinjiang Medical University; \\ ${ }^{2}$ State Key Laboratory of Pathogenesis, Prevention and Treatment of High Incidence Diseases in Central Asia; \\ ${ }^{3}$ Health Management Center, Xinjiang Medical University; ${ }^{4}$ Cancer Hospital Affiliated of Xinjiang Medical University, \\ Urumqi, Xinjiang Uygur Autonomous Region 830000; ${ }^{5}$ Key Laboratory of Cancer Immunotherapy and Radiotherapy, \\ Chinese Academy of Medical Sciences, Beijing 830000, P.R. China
}

Received December 17, 2019; Accepted June 1, 2020

DOI: 10.3892/or.2020.7711

\begin{abstract}
A previous proteomic screening of differentially expressed biomarkers between Kazakh patients with esophageal squamous cell carcinoma (ESCC) and normal adjacent tissues demonstrated that heat shock protein 27 (HSP27) and pyruvate kinase isoenzyme M2 (PKM2) were both highly expressed in ESCC samples compared with normal controls. However, the regulatory association between HSP27 and PKM2 in ESCC remains elusive. In the present study, immunohistochemistry and immunoblotting were adopted to examine the expression of HSP27, PKM2 and other relevant biomarkers involved in epithelial-to-mesenchymal transition in clinical tissue samples. The interactions between proteins were detected by co-immunoprecipitation (Co-IP) assay and further confirmed by immunofluorescence assay. The growth and motility of ESCC cells were examined by MTT, Transwell and wound healing assays. Overexpression of HSP27 was found to be significantly associated with T-cell classification, lymph node metastasis and poor prognosis in ESCC. In addition, HSP27 expression was significantly correlated with PKM2 expression in ESCC specimens. Functionally, knockdown of HSP27 inhibited the growth and motility of ESCC cells. Moreover, HSP27 was found to directly interact with small ubiquitin-related modified protein $2 / 3$ (SUMO2/3) in ESCC cell lines, as evidenced by Co-IP and laser confocal imaging. In addition, downregulation of HSP27 was shown
\end{abstract}

Correspondence to: Dr Xiaomei Lu, Clinical Medical Research Institute, First Affiliated Hospital of Xinjiang Medical University, Room 602, 6th Floor, KEJI Building, 137 Liyushan South Road, Urumqi, Xinjiang Uygur Autonomous Region 830000, P.R. China E-mail: luxiaomei88@163.com

${ }^{*}$ Contributed equally

Key words: esophageal squamous cell carcinoma, heat shock protein 27 , SUMOylation, pyruvate kinase isoenzyme M2 to decrease PKM2 and E-cadherin expression. Knockdown of SUMO2/3 was observed to reduce the expression of HSP27, PKM2 and EMT-related biomarkers. The results of the present study indicated that the SUMOylation of HSP27 enhances the proliferation, invasion and migration of ESCC cells via PKM2.

\section{Introduction}

Esophageal squamous cell carcinoma (ESCC) is one of the most common malignant tumors in China, and its mortality rate ranks fourth $(1,2)$ among the top 10 cancers in China. In particular, the Yili region of Xinjiang is a high-incidence area of ESCC, with the highest incidence observed in the Kazakh population, reaching $150 / 100,000$, with a 5-year survival rate of only $10-13 \%(3,4)$. Therefore, identifying malignant phenotype-related targets and elucidating the molecular regulatory mechanisms involved in the progression of ESCC is crucial for accurate diagnosis, timely individualized treatment and improvement of the survival rate of ESCC.

Pyruvate kinase isoenzyme M2 (PKM2), a rate-limiting enzyme in cellular glycolysis, plays a key role in anaerobic glycolysis (5). Generally, PKM2 exerts tumor-promoting effects via regulation of a number of signaling pathways in tumor cells, operating as a potential protein kinase (6). Our previous study (7) demonstrated that PKM2 was markedly overexpressed in ESCC tissues from Kazakh patients compared with paired adjacent normal tissues through proteomics, suggesting its oncological trait. Our subsequent research on PKM2 (8) and a relevant study by other researchers (9) indicated that upregulated PKM2 may promote the growth and motility of ESCC cells, and that the upregulation of PKM2 in Kazakh patients with ESCC is closely associated with shorter overall survival. However, the molecular mechanism underlying the role of PKM2 in Kazakh patients with ESCC remains elusive.

Heat shock proteins (HSPs) are a group of highly conserved molecular chaperones, mainly comprising HSP27, HSP60, HSP70 and HSP90 (10). Although the relative molecular mass of each of the main HSP groups is distinct, they share similar 
or identical conserved domains (11), and play multiple roles in cell functions, including protein folding, assembly and degradation (12). HSP27 has been reported to be commonly upregulated in several different types of tumors, including ESCC, and is important in the regulation of cancer cell proliferation, apoptosis and metastasis (13-15). Additionally, it also has important biological functions in the regulation of various carcinogenic proteins (16). However, few studies have described the regulatory association between HSP27 and PKM2 in ESCC. By contrast, one recent study reported that HSP90 could stabilize and regulate PKM2 abundance, thereby accelerating anaerobic glycolysis in hepatocellular carcinoma (HCC) (7), which suggests that HSP27 may also interact with PKM2.

Small ubiquitin-related modified proteins (SUMOs) are a class of small protein molecules (17). There are four SUMO homologues in the human genome, namely SUMO1, SUMO2, SUMO3 and SUMO4. SUMO2 and SUMO3 currently cannot be distinguished by antibodies, as they have $\sim 97 \%$ sequence homology except for a difference of two amino acids, and they are collectively referred to as SUMO2/3 (18). An important post-translational modification involving SUMO proteins, which has been termed SUMOylation, is involved in the modulation of various cellular processes, including transcriptional regulation, subcellular localization and target protein stability (19). Extensive research has confirmed that SUMOylation plays a crucial role in human diseases (20). It was recently reported that HSP27 and SUMO2/3 combine directly, which induces SUMOylation of HSP27 to accelerate the invasion and metastasis of HCC cells (21). However, it remains unclear whether there is an interaction between HSP27 and SUMO2/3 in ESCC.

In the present study, based on the findings of previous research, it was hypothesized that HSP27 could physically bind with SUMO2/3, thereby upregulating PKM2 in ESCC cells. To test this hypothesis, experiments were conducted on ESCC specimens and cell lines. Immunohistochemistry (IHC) was performed to examine whether the expression of HSP27 was associated with local infiltration, lymph node metastasis and prognosis. Moreover, the association between HSP27 and PKM2 in ESCC tissues was investigated. The aim of the present study was to elucidate the effects of HSP27 on the growth and motility of ESCC cells, to determine whether SUMOylation of HSP27 can promote the malignant behavior of ESCC cells, and whether this effect is mediated by regulating PKM2 expression.

\section{Materials and methods}

Pathological tissue specimens. The present study was approved by the Medical Ethics Committee of the First Affiliated Hospital of Xinjiang Medical University, and informed consent was obtained from each participant involved. There were 180 ESCC samples on the tissue microarray chip (HEso-Squ180Sur-04, Outdo Biotech), including 100 cancer tissue specimens and 80 normal adjacent tissues (NATs). A chip with 165 samples of Kazakh ESCC tissues from the First Affiliated Hospital of Xinjiang Medical University included 99 ESCC tissues and 57 NATs. None of the patients had received anticancer treatment prior to surgical resection. All cases were diagnosed and confirmed histopathologically. The stages and classifications were graded in accordance with the 2017 WHO classification and grading system (22).

IHC staining. The tissue microarray was dewaxed, hydrated, treated with EDTA antigen repair solution (Sigma-Aldrich; Merck KGaA) and blocked in serum (Zhongshanjinqiao) for $1 \mathrm{~h}$ at room temperature. Endogenous peroxidase activity was blocked with $3 \% \mathrm{H}_{2} \mathrm{O}_{2}$. Subsequently, the tissues were probed at $4^{\circ} \mathrm{C}$ overnight with primary antibodies targeting HSP27 (1:200, ab12351, Abcam) and PKM2 (1:100, 15822-1-AP, ProteinTech Group, Inc.), followed by addition of the secondary antibody (KIT-5030, MXB Biotech) with incubation at $37^{\circ} \mathrm{C}$ for $1 \mathrm{~h}$, and staining using a DAB kit (ZLI-9018, OriGene Technologies, Inc.) for $2 \mathrm{~min}$ at room temperature. The immunostaining was scored by a pathological image analysis software processing system (ImageJ software, version 1.50; National Institutes of Health). According to the comprehensive analysis of staining intensity ( 0 , none; 1 , weak; 2 , moderate; and 3 , strong) and percentage of stained cells $(0,<5 \%$; $1,5-25 \% ; 2,25-50 \%$; and 3,>50\%), each specimen was graded using a total score derived from adding the staining intensity and percentage scores. Scores $<2$ were defined as negative and $>2$ as positive.

Cell culture. The KYSE30, KYSE150, KYSE450 and KYSE510 cells were kind gifts from Professor Zhihua Liu (Chinese Academy of Sciences, Beijing, China); and TE-1, Eca109 and EC9706 cells were purchased from Wuhan University, China. All the cells were maintained in RPMI-1640 medium (Gibco; Thermo Fisher Scientific, Inc.) supplemented with 10\% FBS (Gibco; Thermo Fisher Scientific, Inc.) and placed at $5 \% \mathrm{CO}_{2}$ and $37^{\circ} \mathrm{C}$.

Lentivirus transfection. ESCC cells were plated in 6-well plates ( $2 \times 10^{5} /$ well) with $20 \mu \mathrm{l}$ lentivirus (Lv; $1 \times 10^{8} \mathrm{TU} / \mathrm{ml}$; Genechem Co., Ltd.), $40 \mu 1$ HiTrans transfection reagent (Genechem Co., Ltd.) and $940 \mu \mathrm{l}$ RPMI-1640 medium (10\% FBS) at 5\% $\mathrm{CO}_{2}$ and $37^{\circ} \mathrm{C}$ for $18 \mathrm{~h}$. The infected cells were sorted by flow cytometry and evaluated by western blotting. HSP27 shRNA (Lv-shRNA-HSP27-1, Lv-shRNA-HSP27-2 and LV-shRNA-HSP27-3) and SUMO2/3 shRNA were obtained from Shanghai GeneChem Co., Ltd. The shRNA target sequences were as follows: shHSP27-1: 5'-GATGAGACT GCCGCCAAGT-3'; shHSP27-2: 5'-CGGACGAGCTGACGG T-CAA-3'; shHSP27-3: 5'-CAGTGGTTAGGCGGCAGCA-3'; and scramble: 5'-TTCTCCGAACGT-GTCACGT-3'.

Western blotting. The total proteins (on ice) were extracted with RIPA lysis buffer (Beijing Solarbio Science \& Technology Co., Ltd.), and the concentration of protein extracted was calculated by a BCA kit. The proteins were separated via $12 \%$ SDS-PAGE and then transblotted to PVDF membranes. Then, they were incubated with $0.5 \%$ skimmed milk for $2 \mathrm{~h}$ at room temperature and the following primary antibodies overnight at $4^{\circ} \mathrm{C}$ : HSP27 (1:800, cat. no. ab12351, Abcam), PKM2 (1:1,000, cat. no. 15822-1-AP, ProteinTech Group, Inc.), E-cadherin (1:1,000, cat. no. 20874-1-AP, ProteinTech Group, Inc.), 
vimentin (1:1,000, cat. no. 10366-1-AP, ProteinTech Group, Inc.), SUMO2/3 (1:800, cat. no. ab190757, Abcam), and GAPDH (1:500, cat. no. 20874-1-AP, ProteinTech Group, Inc.). A western blot assay kit (WB7105, Thermo Fisher Scientific, Inc.) provided the secondary antibody, which was applied at room temperature for $1 \mathrm{~h}$, and then chromogen (supplied in the kit) was applied for 5 min in the dark.

MTT assay. A total of 5,000 cells per well were seeded in a 96-well plate. Cells were cultured in RPMI-1640 medium containing $10 \%$ FBS. Detection was conducted at 0, 24, 48, 72 and $96 \mathrm{~h}$. Each group was detected in quintuplicate at each time point. MTT (5 mg/ml, Sigma-Aldrich; Merck KGaA) was added to each well at $20 \mu \mathrm{l} /$ well for $4 \mathrm{~h}$ in the dark, after which time the liquid was discarded and DMSO (135 $\mu \mathrm{l} /$ well, Sigma-Aldrich; Merck KGaA) was added for $10 \mathrm{~min}$ in the dark to dissolve the crystals. The absorbance was measured at $490 \mathrm{~nm}$ by an enzyme labelling instrument (Benchmark ${ }^{\mathrm{TM}}$ Microplate Reader; Bio-Rad Laboratories, Inc.).

Transwell assay. Transwell chambers (24-well; Corning, Inc.) with a $5-\mu \mathrm{m}$ pore filter were coated with a mixture of $60 \mathrm{ml}$ 1:9 Matrix (BD Biosciences) and serum-free RPMI-1640 medium to form a matrix barrier. Precoating was performed at $37^{\circ} \mathrm{C}$ for $1 \mathrm{~h}$ and the Transwell inserts were refrigerated at $4^{\circ} \mathrm{C}$ overnight. Before inoculation, RPMI-1640 medium was used to hydrate the basement membrane for $30 \mathrm{~min}$ at $37^{\circ} \mathrm{C}$. The upper chamber cells were filled with $200 \mu \mathrm{l}$ serum-free RPMI-1640 medium, and the lower chamber was filled with $500 \mu 1$ RPMI-1640 medium supplemented with $10 \% \mathrm{FBS}$. Following incubation at $37^{\circ} \mathrm{C}$ for $48 \mathrm{~h}$, the cells were washed twice with PBS ( 5 min per wash) and fixed with $600 \mu \mathrm{l}$ polyformaldehyde for $15 \mathrm{~min}$ at $4^{\circ} \mathrm{C}$. The non-invading cells were wiped with a cotton swab, washed with $\mathrm{ddH}_{2} \mathrm{O}$ three times and stained with $5 \%$ crystal violet solution for $5 \mathrm{~min}$ at room temperature (23). The number of invading cells was counted in four random fields using an inverted phase contrast microscope (Olympus Corporation) at a $\times 50$ magnification.

Wound healing assay. Cells were seeded in a 6-well cell culture plate (Corning, Inc.) and cultured in serum-free medium for $24 \mathrm{~h}$ at room temperature. After the cells had reached a confluence of $>80 \%$, three parallel scratches were created with a $10-\mu 1$ pipette tip in each hole. Images were captured using an inverted phase contrast microscope (Olympus Corporation) at 0 and $24 \mathrm{~h}$ after scratching. Cell migration was quantitated by measuring the blank area between the moving cell boundaries using Image $\mathrm{J}$ software, version 1.50 (National Institutes of Health).

Co-immunoprecipitation (Co-IP). Co-IP was carried according to the instructions included in the assay kit (Pierce 26149, Thermo Fisher Scientific, Inc.). First, the total protein lysate was extracted, and $500 \mu \mathrm{g}$ total protein was mixed with $2 \mu \mathrm{g}$ monoclonal antibody or IgG and oscillated for $4 \mathrm{~h}$ at $4^{\circ} \mathrm{C}$. The protein beads in the kit were added to the mixture and oscillated overnight at $4^{\circ} \mathrm{C}$. Subsequently, the beads were washed using IP buffer three times. Sample buffer (5X) was mixed with the beads and boiled for $5 \mathrm{~min}$. The supernatant was prepared for western blot analysis.
Immunofluorescence. Cells for immunofluorescence were fixed with $4 \%$ paraformaldehyde at $4^{\circ} \mathrm{C}$ for $20 \mathrm{~min}$ and then subjected to treatment with $0.5 \%$ Triton X-100 diluted with PBS at room temperature for $30 \mathrm{~min}$. Cells were blocked in PBS with $2 \%$ BSA (Sigma-Aldrich; Merck KGaA) for $30 \mathrm{~min}$ at $37^{\circ} \mathrm{C}$ and incubated with antibodies specific for HSP27 (1:200, cat. no. 66767-1-Ig, ProteinTech Group Inc.) and SUMO2/3 (1:200, cat. no. ab190757, Abcam) overnight at $4^{\circ} \mathrm{C}$. Alexa Fluor-conjugated secondary antibodies (Alexa Fluor 488, cat. no. ab150077, Abcam and Alexa Fluor 594, cat. no. ab150120, Abcam) were incubated for $2 \mathrm{~h}$ at room temperature in the dark. Hoechst 33342 was then used for counterstaining the nucleus for $15 \mathrm{~min}$ at room temperature, and images were captured by a laser scanning confocal microscope at a magnification of x200 (LSM710, Carl Zeiss AG).

Statistical analysis. SPSS 19.0 (IBM Corp.) was used to analyze and process the data. The data are expressed as the mean \pm standard deviation. Student's t-test or one-way ANOVA with Bonferroni analysis were used to evaluate the differences among groups. Cross-table test and Pearson's correlation analysis were adopted to analyze the correlation between HSP27 and PKM2. The Kaplan-Meier method was employed to analyze overall survival data. $\mathrm{P}<0.05$ was considered to indicate statistically significant differences.

\section{Results}

Overexpression of HSP27 is associated with ESCC progression and adverse outcomes. To investigate whether HSP27 is involved in the pathological process of ESCC, 224 ESCC tissues and 121 NATs were collected. HSP27 expression was detected by IHC, which revealed that HSP27 was markedly overexpressed in cancer tissues compared with control tissues (Fig. 1A, Table I). The association between HSP27 level and pathological information was analyzed; Table I shows that HSP27 was significantly correlated with $\mathrm{T}$ classification and lymph node metastasis. However, there was no marked correlation with other pathological information, including demographic characteristics, tumor size, pathological grade and pathological classification. Kaplan-Meier survival analysis revealed that the survival of ESCC patients with high HSP27 expression was markedly inferior to that of those with low HSP27 expression (Fig. 1B, $\mathrm{P}<0.05$ ). In addition, to further confirm the correlation between HSP27 and PKM2 in ESCC tissues, the expression of PKM2 and HSP27 expression was next investigated in $165 \mathrm{Kazakh}$ ESCC tissues by IHC staining. The results demonstrated that both HSP27 and PKM2 were upregulated in Kazakh ESCC tissues compared with NATs (Fig. 1A and C). The subcellular localization of HSP27 and PKM2 was mainly cytoplasmic, with that of PKM2 being nucleoplasmic in a few cases, in accordance with the positive staining indicated by the primary antibodies used. Furthermore, the $\chi^{2}$ test $\left(\chi^{2}=4.797, P=0.029\right.$, Table II) and Spearman's correlation analysis $(r=0.253, P=0.0011$, Fig. 1D) revealed that HSP27 expression was significantly correlated with PKM2.

HSP27 promotes the growth and motility of ESCC cells. Next, to explore the biological roles of HSP27 in the growth and 
A

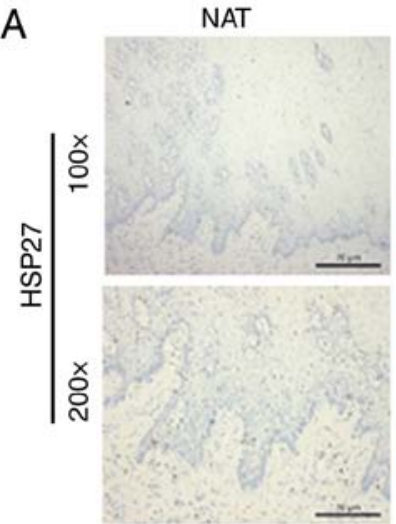

C

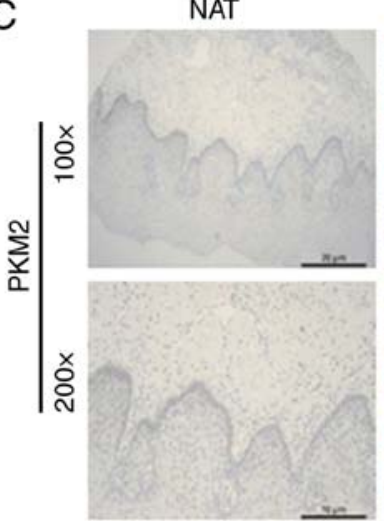

ESCC

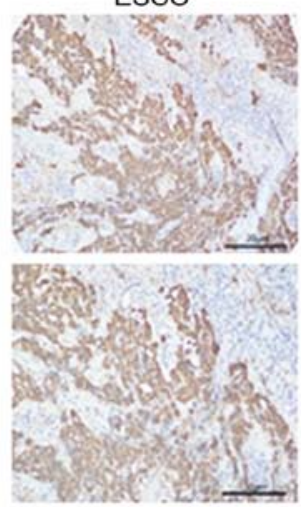

ESCC

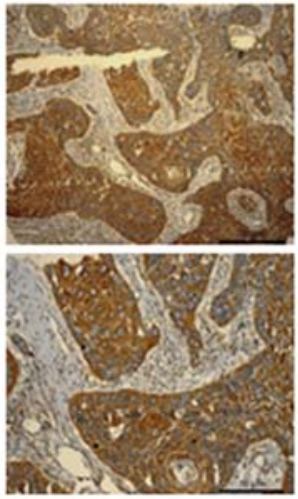

B
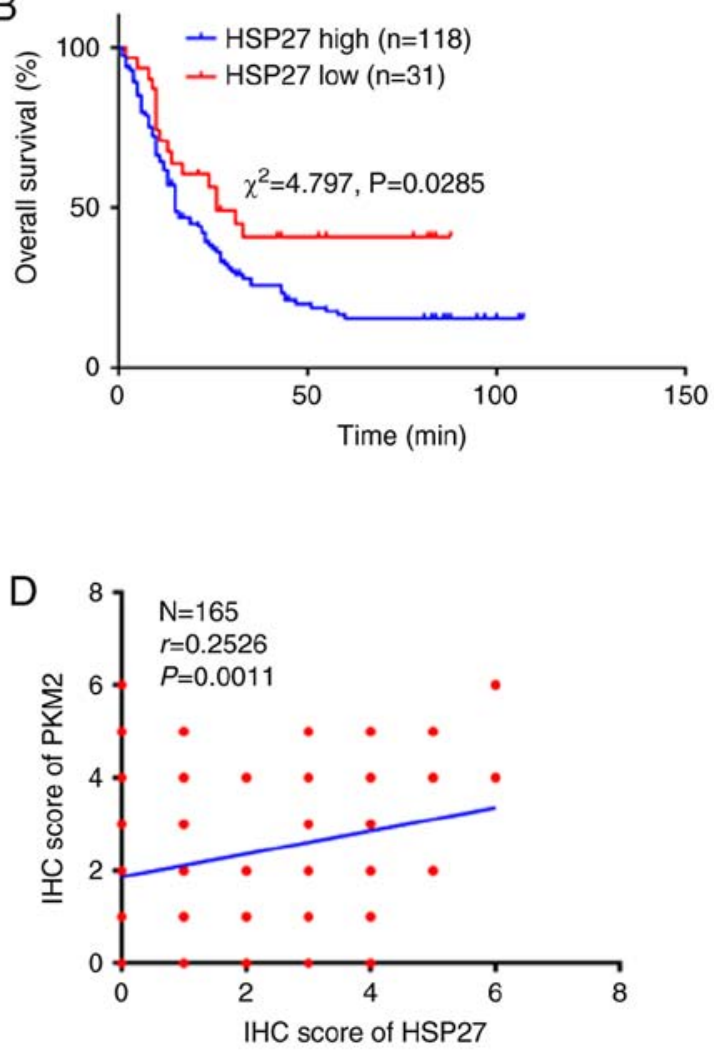

Figure 1. HSP27 is markedly upregulated in ESCC and is correlated with PKM2 expression and poor prognosis. (A) IHC results of HSP27 expression in ESCC tissues and NAT (magnification, x100 and x200). (B) Survival curve of HSP27 expression in ESCC. (C) IHC results of PKM2 in ESCC tissues and NAT (magnification, $x 100$ and x200). (D) Spearman's correlation analysis between HSP27 and PKM2 expression in ESCC. Representative selected images are shown. The log-rank test was used in survival analysis $\left(\chi^{2}=4.797, \mathrm{P}=0.0285\right)$, and Spearman's method was employed to analyze the correlation $(\mathrm{N}=165$, $\mathrm{r}=0.2526, \mathrm{P}=0.0011)$. ESCC, esophageal squamous cell carcinoma; HSP, heat shock protein; PKM2, pyruvate kinase isoenzyme 2; IHC, immunohistochemistry; NAT, normal adjacent tissue.

invasion of ESCC, HSP27 expression in seven ESCC cell lines (Eca109, EC9706, KYSE30, KYSE450, TE-1, KYSE150 and KYSE510) was evaluated using immunoblotting; among these cell lines, KYSE450 exhibited the highest level of HSP27 expression, while KYSE150 had the second-highest HSP70 level (Fig. 2A). To determine the biological function of HSP27 in ESCC, Lv-based shRNA targeting HSP27 was transfected into KYSE450 and KYSE150 cells. Stable HSP27 knockdown cell lines were established successfully and confirmed by western blotting (Fig. 2B). Next, the absorbance of the HSP27 knockdown group was reduced significantly compared with that of the control group in KYSE450 cells with the extension of time in the MTT assay (Fig. 2C). KYSE150 cells exhibited a similar trend in the MTT assay: The absorbance of the HSP27 knockdown group was significantly reduced at 72 and 96 h (Fig. 2D). MTT data supported that HSP27 expression was correlated with the proliferation of ESCC cells. Subsequently, the Transwell assay was used to evaluate the invasion ability of KYSE450 and KYSE150 cells with HSP27 knockdown. Compared with the control groups, the number of cells invading through the cell membrane in the HSP27 knockdown group significantly decreased after $48 \mathrm{~h}(\mathrm{P}<0.05$, Fig. $2 \mathrm{E}$ and $\mathrm{F})$, indicating that HSP27 promoted the invasiveness of ESCC cells. As shown by the wound healing assay, the migration of KYSE450 and KYSE150 cells was reduced by HSP27 knockdown $(\mathrm{P}<0.05$,
Fig. 2G and H). Therefore, HSP27 can accelerate the growth and increase the motility of ESCC cells.

HSP27 promotes ESCC cell function by regulating PKM2. To understand whether HSP27 enhanced the growth and motility of ESCC cells via PKM2, the expression of PKM2 and other biomarkers was detected by immunoblotting. The expression of PKM2 and vimentin was reduced after silencing HSP27; conversely, E-cadherin expression was markedly increased (Fig. 3A). To determine whether there is a direct interaction between PKM2 and HSP27, Co-IP was conducted. The results of Co-IP revealed no direct interaction between HSP27 and PKM2 (Fig. 3B).

HSP27 regulates the expression of PKM2 by interacting with $S U M O 2 / 3$. The molecular mechanism through which HSP27 regulates PKM2 expression was further investigated. Lv-packaged SUMO2/3 shRNAs were transfected into KYSE450 and KYSE150 cells. The results of western blotting demonstrated that SUMO2/3 was successfully knocked down, so the cells were used for subsequent studies (Fig. 4A and B). Subsequently, immunoblotting was used to detect alterations in HSP27, PKM2 and EMT-related molecules. The expression of HSP27, PKM2 and the EMT-related biomarker vimentin was significantly decreased, whereas the expression 
Table I. Clinicopathological significance of HSP27 expression in ESCC.

\begin{tabular}{|c|c|c|c|c|c|}
\hline \multirow[b]{2}{*}{ Characteristics } & \multirow[b]{2}{*}{$\mathrm{N}$} & \multicolumn{2}{|c|}{ HSP27 expression } & \multirow[b]{2}{*}{$\chi^{2}$} & \multirow[b]{2}{*}{ P-value } \\
\hline & & High & Low & & \\
\hline \multicolumn{6}{|l|}{ Tissue type } \\
\hline ESCC & 224 & 152 & 72 & 18.854 & 0.000 \\
\hline NAT & 121 & 53 & 68 & & \\
\hline Sex & & & & 1.817 & 0.178 \\
\hline Male & 239 & 147 & 92 & & \\
\hline Female & 106 & 57 & 49 & & \\
\hline Age (years) & & & & 0.008 & 0.928 \\
\hline$\leq 60$ & 139 & 83 & 56 & & \\
\hline$>60$ & 206 & 122 & 84 & & \\
\hline Tumor diameter $(\mathrm{cm})$ & & & & 1.34 & 0.247 \\
\hline$<5$ & 200 & 115 & 85 & & \\
\hline$\geq 5$ & 117 & 75 & 42 & & \\
\hline Pathological grade & & & & 2.636 & 0.268 \\
\hline I & 41 & 28 & 13 & & \\
\hline II & 211 & 121 & 90 & & \\
\hline III & 91 & 59 & 32 & & \\
\hline Pathological type & & & & 5.216 & 0.157 \\
\hline Ulcerative & 190 & 111 & 79 & & \\
\hline Medullary & 88 & 50 & 38 & & \\
\hline Protruding & 37 & 23 & 14 & & \\
\hline Stenosing & 7 & 7 & 0 & & \\
\hline $\mathrm{T}$ classification & & & & 16.4 & 0.000 \\
\hline $\mathrm{T} 1-2$ & 86 & 34 & 52 & & \\
\hline T3-4 & 243 & 157 & 86 & & \\
\hline Lymph node metastasis & & & & 3.889 & 0.049 \\
\hline N0 & 204 & 111 & 93 & & \\
\hline N1-N3 & 135 & 88 & 47 & & \\
\hline
\end{tabular}

Bold print indicates statistical significance. HSP, heat shock protein; ESCC, esophageal squamous cell carcinoma; NAT, normal adjacent tissue.

Table II. Correlation between HSP27 and PKM2 expression in ESCC.

\begin{tabular}{lrrrrr}
\hline & & \multicolumn{2}{c}{ HSP27 expression } & & \\
\cline { 3 - 5 } PKM2 expression & $\mathrm{N}$ & High & Low & $\chi^{2}$ & P-value \\
\hline High & 91 & 64 & 27 & 35 & 0.024 \\
Low & 74 & 39 & 62 & \\
Total & 165 & 103 & & \\
\hline
\end{tabular}

HSP, heat shock protein; PKM2, pyruvate kinase isoenzyme 2; ESCC, esophageal squamous cell carcinoma.

of the EMT-related biomarker E-cadherin was increased in both KYSE450 and KYSE150 knockdown cells compared to control cells (Fig. 4C-F). Next, the Co-IP results revealed that HSP27 was capable of directly binding with SUMO 2/3 (Fig. 4D and E). To further verify the association between SUMO2/3 and HSP27, immunofluorescence was used to detect the subcellular localization of HSP27 and SUMO2/3. The laser confocal microscopy images demonstrated that SUMO2/3 and HSP27 were co-localized in the cytoplasm (Fig. 4F). Taken together, these data demonstrated that HSP27 directly interacts with SUMO2/3, thereby regulating PKM2 and promoting the motility of ESCC cells through the EMT pathway. 

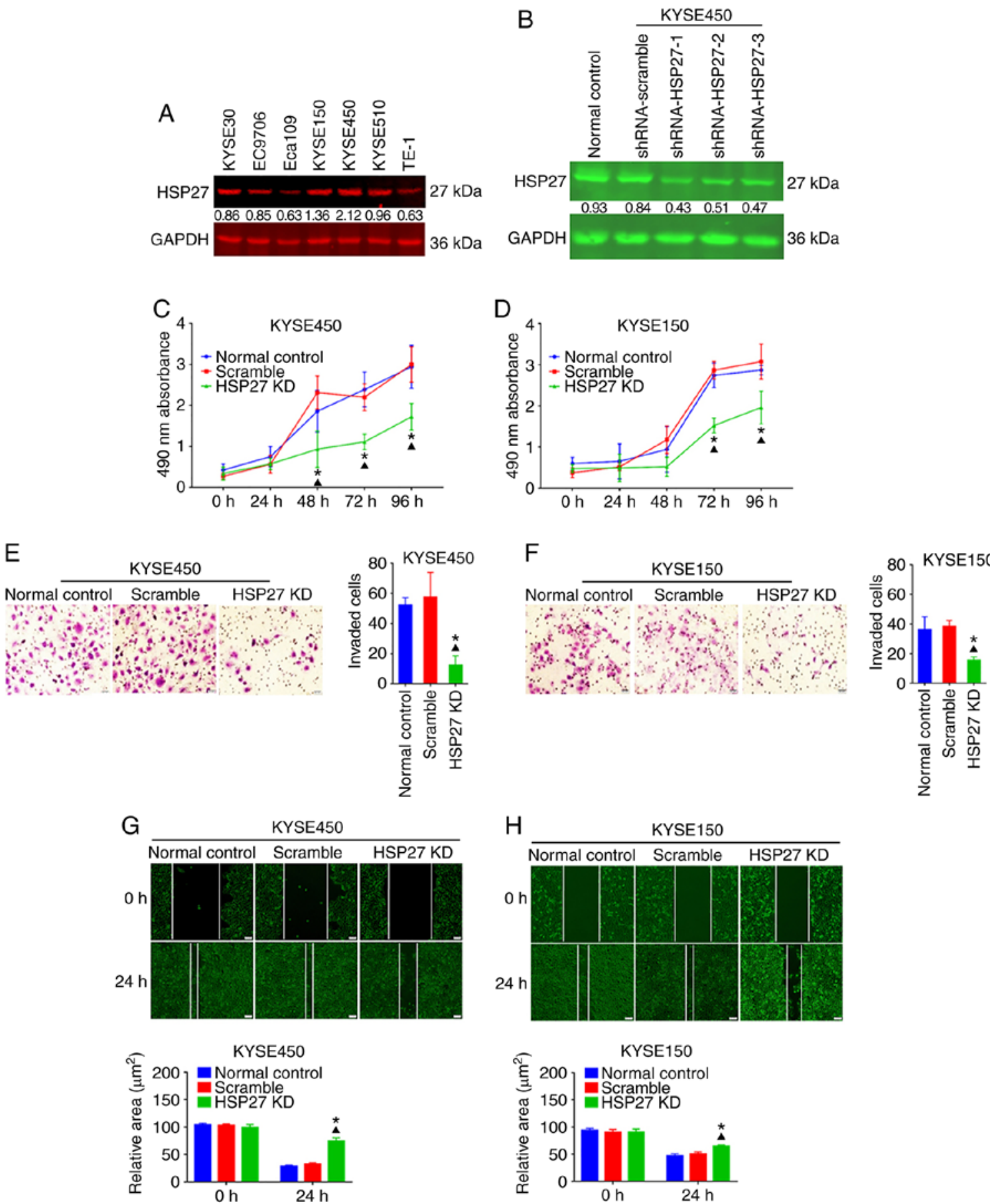

Figure 2. HSP27 promotes the proliferation, invasion and migration of ESCC cells. (A) Basal level of HSP27 expression in a panel of ESCC cell lines, including KYSE30, KYSE150, KYSE450, KYSE510, TE-1, Eca109 and EC9706, as detected by western blotting. (B) Detection of knockdown efficiency of HSP27 shRNA vectors in KYSE450 cells by western blotting. GAPDH was used as an internal loading control. (C and D) MTT assay of proliferation after knockdown of HSP27 in KYSE450 and KYSE150 cells. (E and F) Changes in invasion, detected by the Transwell assay, in KYSE450 and KYSE150 cells after knockdown of HSP27 (magnification, x100). (G and H) Variations in the migratory activity of KYSE450 and KYSE150 cells analyzed using the wound healing assay after knocking down HSP27 (magnification, $x 100$ ). ${ }^{*} \mathrm{P}<0.05$ compared with the normal control group and ${ }^{\mathbf{P}} \mathrm{P}<0.05$ compared with the shRNA-scramble control group. ESCC, esophageal squamous cell carcinoma; HSP, heat shock protein; KD, knockdown.

SUMO2/3 promotes the proliferation and motility of ESCC cells. To elucidate the effects of SUMO2/3 on the malignant behavior of ESCC cells, MTT, wound healing and Transwell assays were performed to assess the proliferation and motility of KYSE450 and KYSE150 cells following SUMO2/3 knockdown. The data suggested that the growth and motility of ESCC cells were significantly suppressed by knocking down SUMO2/3 (Fig. 5), which supported our hypothesis that
SUMOylation of HSP27 regulates the expression of PKM2, promoting the infiltration and metastasis of ESCC cells through the EMT pathway.

\section{Discussion}

Accumulating evidence has revealed that HSPs, as molecular chaperones, play a pivotal role in the modulation of protein 
A

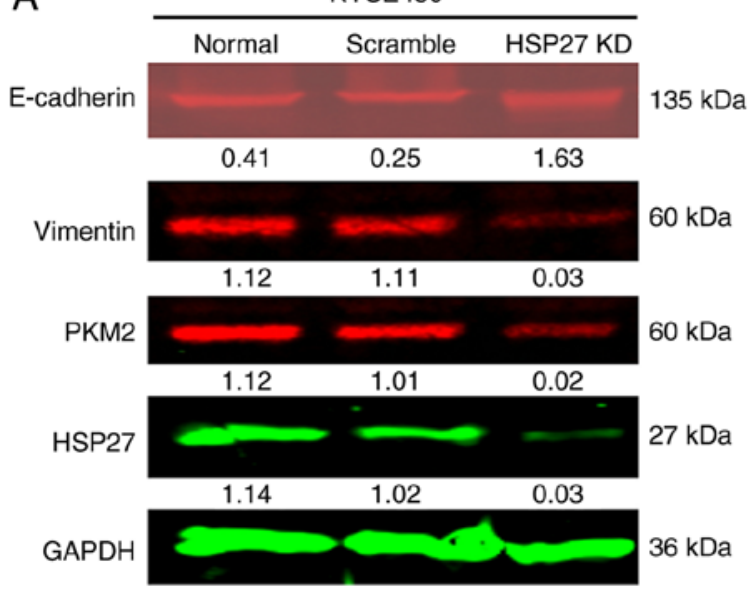

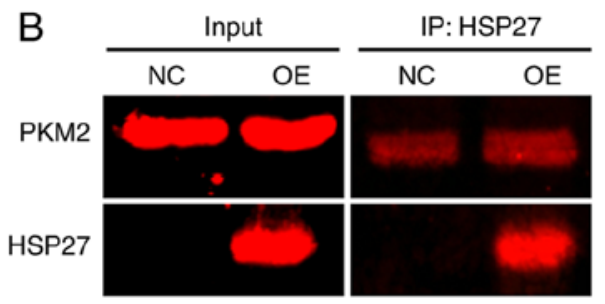

Figure 3. HSP27 modulates but does not interact directly with PKM2 in KYSE450 cells. (A) Western blotting results of downstream proteins (PKM2, vimentin and E-cadherin) following HSP27 knockdown in KYSE450 cells. GAPDH, internal loading control. (B) No interaction between HSP27 and PKM2 was observed in the co-immunoprecipitation assay. HSP, heat shock protein; PKM2, pyruvate kinase isoenzyme 2; KD, knockdown; NC, negative control; $\mathrm{OE}$, overexpression.

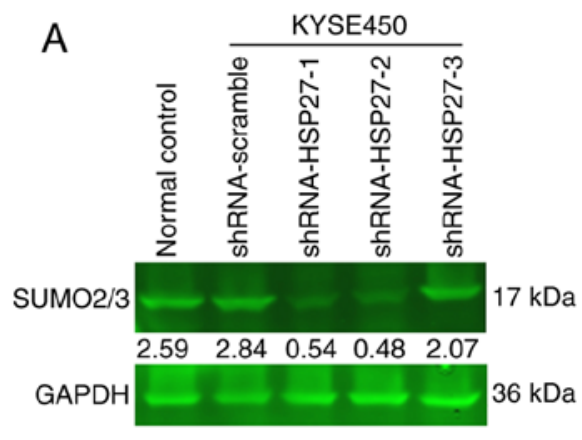

D

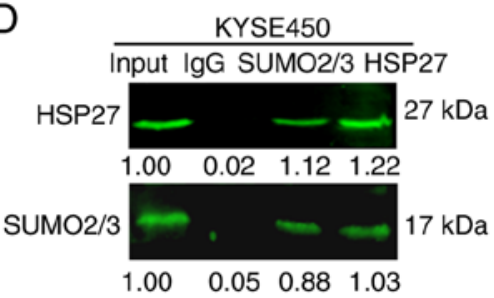

E

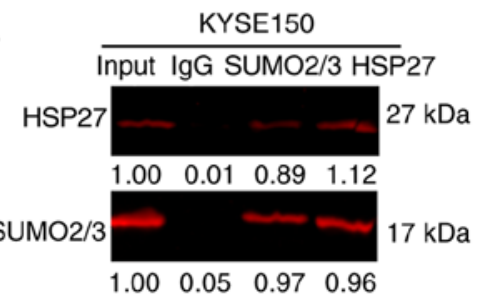

B
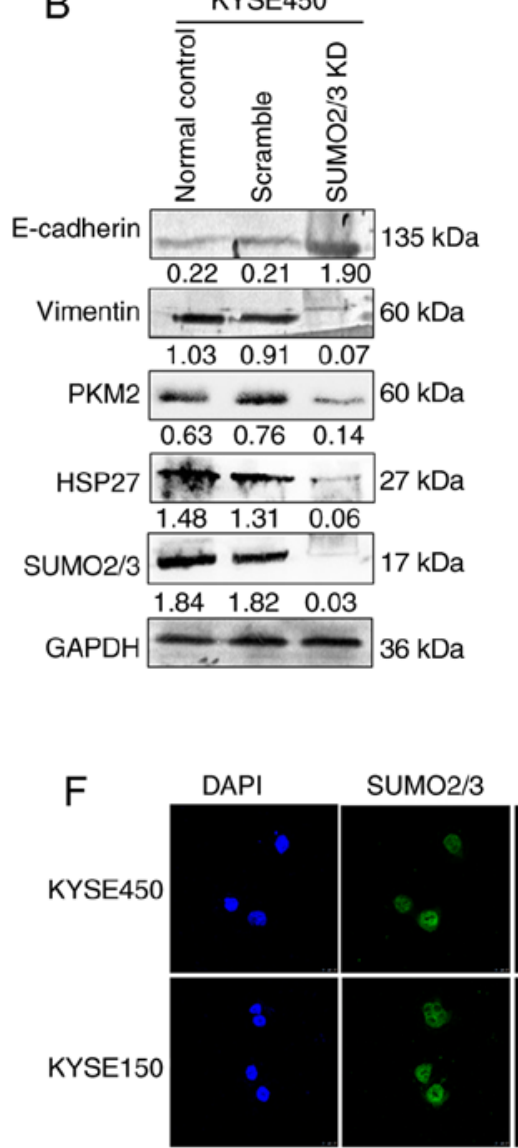
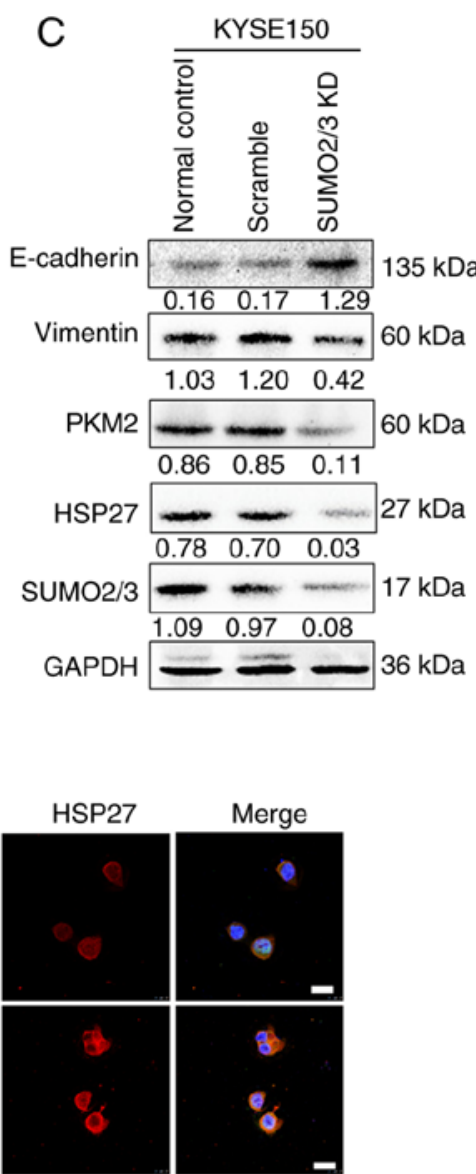

Figure 4. HSP27 regulates PKM2 expression through SUMO2/3 in KYSE450 and KYSE150 cells. (A) Detection of the knockdown efficiency of SUMO2/3 shRNA vectors in KYSE450 cells by western blotting. GAPDH, internal loading control. (B and C) Detection of HSP27, PKM2, vimentin and E-cadherin in KYSE450 and KYSE150 cells after SUMO2/3 silencing by western blotting. (D and E) Interaction between HSP27 and SUMO2/3, as shown by co-immunoprecipitation assay. (F) Immunofluorescence of nuclei (DAPI, blue), SUMO2/3 (green) and HSP27 (red) in KYSE450 and KYSE150 cells. HSP, heat shock protein; PKM2, pyruvate kinase isoenzyme 2; SUMO2/3, small ubiquitin-related modified protein 2/3; KD, knockdown.

conformation and stability, and in carcinogenesis. As an important member of the HSP family, HSP27 has been reported to be highly expressed in different human cancers, including liver, lung and breast cancer, and is involved in drug resistance, cell growth, cell apoptosis, tumorigenesis and metastasis, as well as other functions (22). 

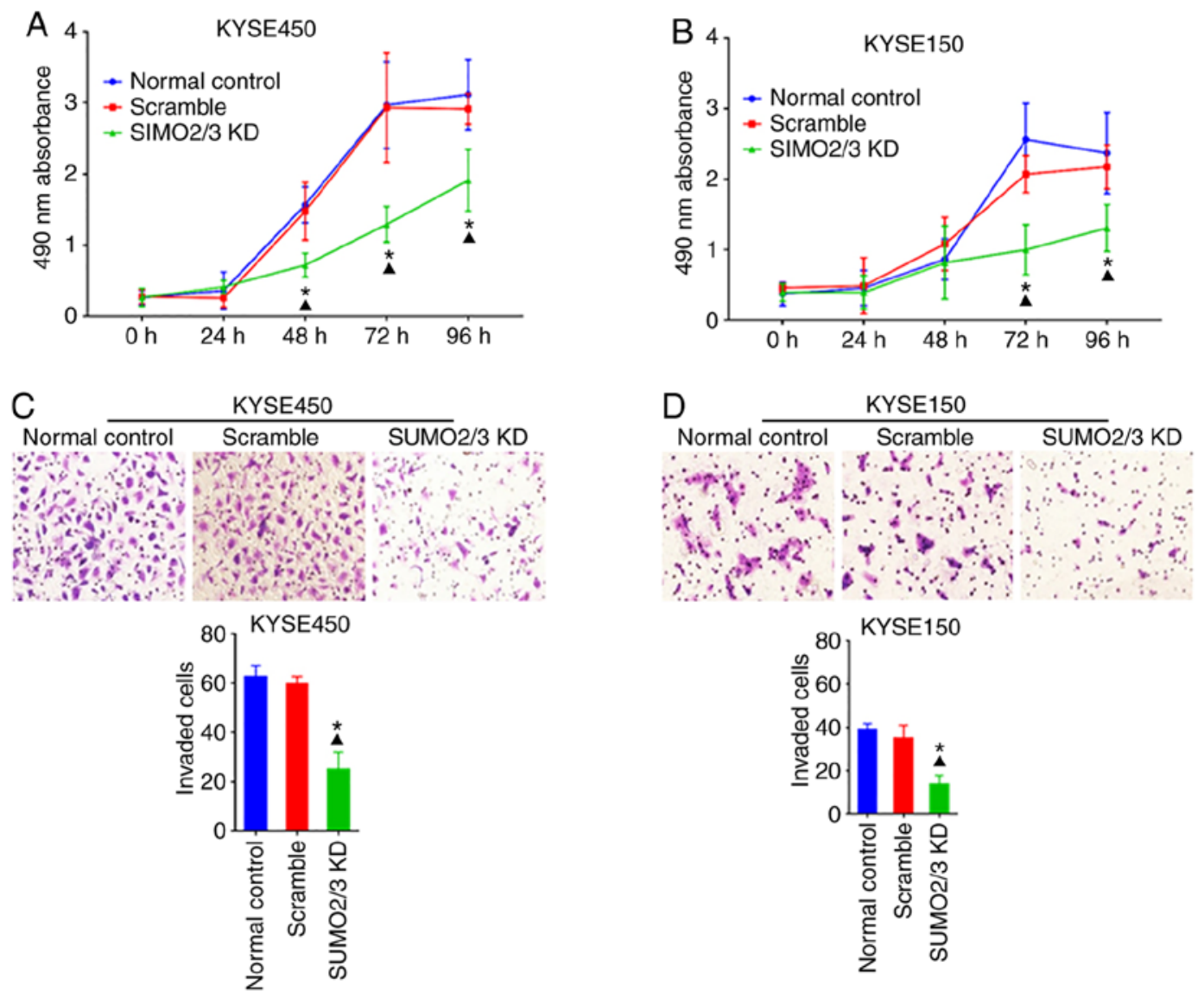

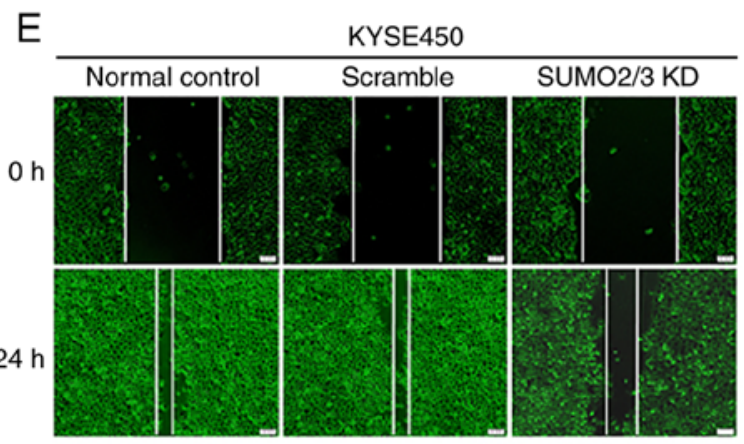

KYSE450

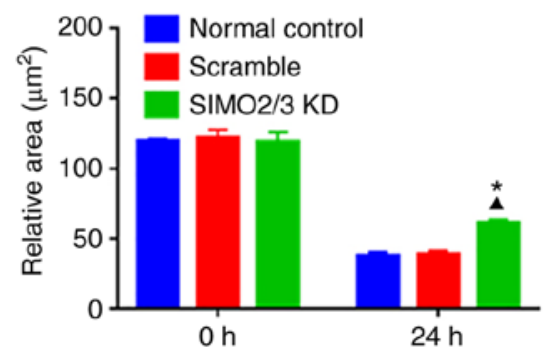

F

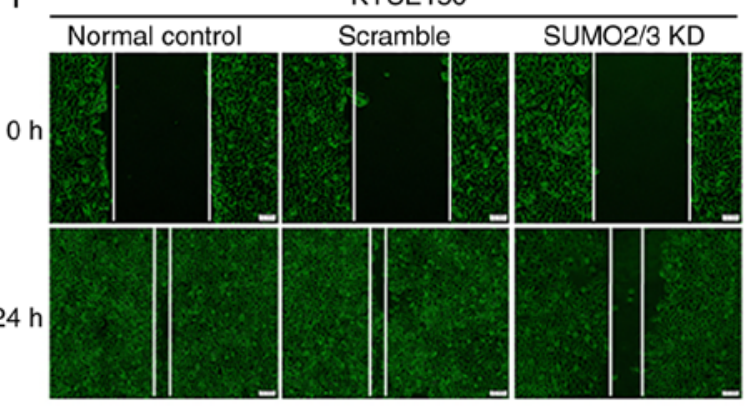

KYSE150

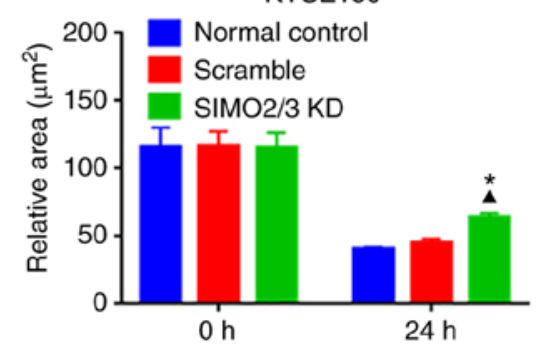

Figure 5. SUMO2/3 promotes ESCC cell proliferation, invasion and migration. (A and B) MTT assay of proliferation changes in KYSE450 and KYSE150 cells after knockdown of SUMO2/3. (C and D) Detection of invasion by Transwell assay in KYSE450 and KYSE150 cells after silencing of SUMO2/3 (magnification, x100). (E and F) Variations in the migratory activity of KYSE450 and KYSE150 cells analyzed using the wound healing assay after SUMO2/3 knockdown (magnification, $\mathrm{x} 100$ ). ${ }^{*} \mathrm{P}<0.05$ compared with the normal control group and ${ }^{\Delta} \mathrm{P}<0.05$ compared with the shRNA-scramble control group. ESCC, esophageal squamous cell carcinoma; HSP, heat shock protein; SUMO2/3, small ubiquitin-related modified protein 2/3; KD, knockdown.

We previously demonstrated using two-dimensional gel electrophoresis that 15 proteins, including HSP27 and PKM2, were highly expressed in Kazakh patients with ESCC (7). In addition, our previous study revealed that the PKM2/STAT3 pathway promoted ESCC cell proliferation and motility through regulating EMT (24). However, the regulatory association between HSP27 and PKM2 remains largely unknown. HSPs are important stress response proteins that protect the stability of the intracellular environment. Whether HSP27 participates in anaerobic glycolysis 
has not been extensively investigated. High expression of HSP90 in myocytes may increase cell uptake and utilization of glucose, and the function of HSP90 depends on the supply of ATP (25), strongly suggesting that HSP90 may directly affect cell glucose metabolism. In healthy cells, upregulation of HSP62 may induce the synthesis of pyruvate kinase, increase the activity of pyruvate kinase, and increase the cell content of ATP, indicating that high expression of HSP62 can accelerate glycolysis in healthy cells (26). It was recently reported that HSP proteins are also involved in the Warburg effect of cancer cells. HSP90 is significantly overexpressed in HCC tissues and can directly bind and phosphorylate threonine 328 of PKM2, stabilizing PKM2 dimers in the cytoplasm and promoting the Warburg effect (27).

In addition to HSP90, HSP40 has also been shown to interact with PKM2. The binding of HSP40 and PKM2 can stabilize the dimer of PKM2, and silencing HSP40 was able to suppress the growth of tumor cells (29). These findings strongly suggest a direct interaction between PKM2 and HSP proteins. HSP27 and HSP90 share highly conserved domains. Therefore, it was hypothesized that HSP27 may also interact with PKM2 directly in Kazakh patients with ESCC. To test this hypothesis, the levels of HSP27 and PKM2 were assessed by IHC in ESCC tissue samples. The staining results demonstrated that the expression of HSP27 was markedly associated with $\mathrm{T}$ stage, lymph node metastasis and overall survival. Moreover, the expression levels of HSP27 and PKM2 were positively correlated in Kazakh ESCC tissues. However, the correlation between HSP27 and SUMO2/3 was not determined in the present study, mainly due to the fact that the expression of SUMO2/3 was not detected at the clinical tissue level. Further verification at the ESCC cell level demonstrated that knockdown of HSP27 inhibited the function of ESCC cells, decreased the expression of PKM2 and vimentin, and increased the expression of E-cadherin. These results indicate that HSP27 regulates the PKM2 pathway to promote ESCC progression. However, the results of Co-IP revealed that HSP27 does not bind directly with PKM2, indicating that HSP27 and PKM2 do not directly interact. Moreover, a previous study by Xu et al (28) on breast cancer reported that knockdown of HSP27 can suppress EMT, with decreased Snail and vimentin and increased E-cadherin expression. These data suggested that HSP27 can promote EMT in breast cancer. Despite the fact that ESCC and breast cancer pathologically originate from different tissues, it may be inferred that HSP27 acts in a similar manner in the two cancers by regulating EMT. EMT variations following knockdown of HSP27 in ESCC cells will be further investigated in future studies.

SUMOylation, an important post-translational modification, is mediated by SUMO2/3 (18). Liu et al (17) found that SUMO2/3 promotes the transformation of chronic hepatitis $\mathrm{B}$ to liver cancer by stabilizing p65 in the cytoplasm and deactivating NF- $\kappa \mathrm{B}$. Ge et al (21) reported that SUMO2/3 in $\mathrm{HCC}$ cells protects HSP27 from degradation by binding to the GKHE sequence on the HSP27 protein, thus promoting the motility of tumor cells. However, the SUMOylation of HSP27 has rarely been reported in ESCC. The data obtained herein demonstrated that SUMOylation of HSP27 was able to modulate the expression PKM2 to promote the progression of ESCC.
In the present study, HSP27 knockdown was shown to downregulate PKM2 in ESCC cells; in addition, the proliferation and invasion of ESCC cells were also markedly suppressed after HSP27 silencing. These data strongly suggest that HSP27 promotes ESCC cell function by regulating PKM2. Mechanistically, the promoting effect of HSP27 on PKM2 level may be mediated by SUMO2/3, which can interact with HSP27. As shown in the present study, silencing of SUMO2/3 may lead to the downregulation of HSP27, along with a decrease in PKM2. From these data, it may be inferred that SUMO2/3 can prevent the HSP27 protein from degradation. These observations were in agreement with a previous study reporting that SUMO2/3 was required for the promotion of proliferation and invasion of liver cancer cells by HSP27 (21). Of note, the data of the present study strongly support that SUMOylation stabilizes HSP27 and protects it from degradation, which was also reported previously by Ge et al (21). However, as the expression of SUMO2/3 was not detected in the present study, no conclusions can be drawn on the correlation between SUMO2/3 and HSP27, which should be further investigated in future studies.

In conclusion, HSP27 was found to be significantly associated with $\mathrm{T}$ stage, lymph node metastasis and poor prognosis in ESCC, revealing a novel regulatory mechanism of PKM2, mediated by SUMOylation of HSP27, which is implicated in the oncogenesis of ESCC.

\section{Acknowledgements}

Not applicable.

\section{Funding}

The present study was funded in part by the Natural Science Foundation of China (grant nos. 81960527, U1603284 and 81860511 ) and in part by Key Research and Development Project of the Xinjiang Uygur Autonamous Region (grant no. 2020B03003-1) and Science and Technology Projects of Xinjiang Uygur Autonomous Region (grant no. 2018E02067) from TianshanXuesong Project of the Xinjiang Uygur Autonomous Region (grant no. 2018XS19).

\section{Availability of data and materials}

The datasets used and/or analyzed during the present study are available from the corresponding author on reasonable request.

\section{Authors' contributions}

XZ and TL performed all the experiments described. STZ drafted and revised the manuscript. QL analyzed and interpreted the patient data obtained from immunohistochemistry. TXS and XJH immunoscored all the sections. QQZ and LFY contributed to the statistical analysis. XML superised the study. All the authors have read and approved the final manuscript.

\section{Ethics approval and consent to participate}

The protocol of the present study was approved by the Medical Ethics Committee of the First Affiliated Hospital of Xinjiang 
Medical University, and informed consent was obtained from each participant involved regarding the use of their tissues for research purposes.

\section{Patient consent or publication}

Not applicable.

\section{Competing interests}

All the authors declare that they have no competing interests.

\section{References}

1. Chen W, Zheng R, Baade PD, Zhang S, Zeng H, Bray F, Jemal A, $\mathrm{Yu}$ XQ and He J: Cancer statistics in China, 2015. CA Cancer J Clin 66: 115-132, 2016.

2. Liu Y, Liu J, Yin P, Liu S, Cai Y, You J, Zeng X, Wang L and Zhou M: The disease burden of malignant tumor in China, 1990 and 2010. Zhonghua Yu Fang Yi Xue Za Zhi 49: 309-314, 2015 (In Chinese)

3. Zheng S, Vuitton L, Sheyhidin I, Vuitton DA, Zhang Y and Lu X: Northwestern China: A place to learn more on oesophageal cancer. Part one: Behavioural and environmental risk factors. Eur J Gastroenterol Hepatol 22: 917-925, 2010.

4. Lu XM, Monnier-Benoit S, Mo LZ, Xu SY, Prétet JL, Liu Z, Vuitton DA and Mougin C: Human papillomavirus in esophageal squamous cell carcinoma of the high-risk Kazakh ethnic group in Xinjiang, China. Eur J Surg Oncol 34: 765-770, 2008.

5. Liu F, Ma F, Wang Y, Hao L, Zeng H, Jia C, Wang Y, Liu P, Ong IM, Li B, et al: PKM2 methylation by CARM1 activates aerobic glycolysis to promote tumorigenesis. Nat Cell Biol 19: 1358-1370, 2017.

6. Wong N, Ojo D, Yan J and Tang D: PKM2 contributes to cancer metabolism. Cancer Lett 356 (2 Pt A): 184-191, 2015.

7. Liu Z, Feng JG, Tuersun A, Liu T, Liu H, Liu Q, Zheng ST, Huang CG, Lv GD, Sheyhidin I and Lu XM: Proteomic identification of differentially-expressed proteins in esophageal cancer in three ethnic groups in Xinjiang. Mol Biol Rep 38: 3261-3269, 2011

8. Liu Q, Liang M, Liu T, Vuitton L, Zheng S, Gao X, Lu M, Li X, Sheyhidin I and Lu X: M2 isoform of pyruvate kinase (PKM2) is upregulated in Kazakh's ESCC and promotes proliferation and migration of ESCC cells. Tumour Biol 37: 2665-2672, 2016.

9. Abdel-Haleem AM, Lewis NE, Jamshidi N, Mineta K, Gao X and Gojobori T: The emerging facets of non-cancerous warburg effect. Front Endocrinol (Lausanne) 8: 279, 2017.

10. Wu J, Liu T, Rios Z, Mei Q, Lin X and Cao S: Heat shock proteins and cancer. Trends Pharmacol Sci 38: 226-256, 2017.

11. Saini J and Sharma PK: Clinical, prognostic and therapeutic significance of heat shock proteins in cancer. Curr Drug Targets 19: 1478-1490, 2018.

12. Zininga T, Ramatsui L and Shonhai A: Heat shock proteins as immunomodulants. Molecules 23: 2846, 2018.

13. Han L, Jiang Y, Han D and Tan W: Hsp27 regulates epithelial mesenchymal transition, metastasis and proliferation in colorectal carcinoma. Oncol Lett 16: 5309-5316, 2018.
14. Zhang $\mathrm{Y}$, Feng Z, Wang W, Dong J, Gong X, Pu H and Chen X: Expression of heat shock protein-27 (Hsp27) and P38MAPK in esophageal squamous cell carcinoma. Med Sci Monit 23: 5246-5253, 2017.

15. Luz CC, Noguti J, Borges de Araujo L, Gianni MS, Simao Gomes T and Ricardo AN: Hsp27 and Hsp70 expression in esophageal squamous. Asian Pac J Cancer Prev 18: 789-794, 2017.

16. Lee MS, Lee J, Lee S, Yoo SM, Kim JH, Kim WT, Kim WJ and Park J: Clinical, prognostic, and therapeutic significance of heat shock protein 27 in bladder cancer. Oncotarget 9: 7961-7974, 2018.

17. Liu J, Sha M, Wang Q, Ma Y, Geng X, Gao Y, Feng L, Shen Y and Shen Y: Small ubiquitin-related modifier $2 / 3$ interacts with $\mathrm{p} 65$ and stabilizes it in the cytoplasm in HBV-associated hepatocellular carcinoma. BMC Cancer 15: 675, 2015.

18. Eifler K and Vertegaal ACO: SUMOylation-mediated regulation of cell cycle progression and cancer. Trends Biochem Sci 40: 779-793, 2015.

19. Princz A and Tavernarakis N: SUMOylation in Neurodegenerative Diseases. Gerontology 66: 122-130, 2020.

20. Dasso M: Biochemistry: Rear view of an enzyme. Nature 497: 576-577, 2013.

21. Ge H, Du J, Xu J, Meng X, Tian J, Yang J and Liang H: SUMOylation of HSP27 by small ubiquitin-like modifier $2 / 3$ promotes proliferation and invasion of hepatocellular carcinoma cells. Cancer Biol Ther 18: 552-559, 2017.

22. Pellat A and Coriat R: Well differentiated grade 3 neuroendocrine tumors of the digestive tract: A narrative review. J Clin Med 9: E1677, 2020.

23. Zhang N, Ren Y, Wang Y, Zhao L, Wang B, Ma N, Gao Z and Cao B: LRG1 suppresses migration and invasion of esophageal squamous cell carcinoma by modulating epithelial to mesenchymal transition. J Cancer 11: 1486-1494, 2020.

24. Ma R, Liu Q, Zheng S, Liu T, Tan D and Lu X: PKM2-regulated STAT3 promotes esophageal squamous cell carcinoma progression via TGF- $\beta 1$-induced EMT. J Cell Biochem 12 Feb, 2019 (Epub ahead of print).

25. Lang BJ, Guerrero-Gimenez ME, Prince TL, Ackerman A, Bonorino $\mathrm{C}$ and Calderwood SK: Heat shock proteins are essential components in transformation and tumor progression: Cancer cell intrinsic pathways and beyond. Int J Mol Sci 20: 4507, 2019.

26. Marsden M, Nickells RW, Kapoor M and Browder LW: The induction of pyruvate kinase synthesis by heat shock in Xenopus laevis embryos. Dev Genet 14: 51-57, 1993.

27. Yang Z, Yang C, Xiao L, Liao X, Lan A, Wang X, Guo R, Chen P, $\mathrm{Hu} \mathrm{C}$ and Feng J: Novel insights into the role of HSP90 in cytoprotection of $\mathrm{H} 2 \mathrm{~S}$ against chemical hypoxia-induced injury in H9c2 cardiac myocytes. Int J Mol Med 28: 397-403, 2011.

28. Xu Q, Tu J, Dou C, Zhang J, Yang L, Liu X, Lei K, Liu Z, Wang Y, Li L, et al: HSP90 promotes cell glycolysis, proliferation and inhibits apoptosis by regulating PKM2 abundance via Thr-328 phosphorylation in hepatocellular carcinoma. Mol Cancer 16: $178,2017$.

29. Huang L, Yu Z, Zhang T, Zhao X and Huang G: HSP40 interacts with pyruvate kinase $\mathrm{M} 2$ and regulates glycolysis and cell proliferation in tumor cells. PLoS One 9: e92949, 2014.

(i) (9) This work is licensed under a Creative Commons Attribution-NonCommercial-NoDerivatives 4.0 International (CC BY-NC-ND 4.0) License. 